\title{
COMO OS NATIVOS AMERICANOS TERIAM UTILIZADO SEUS ESPELHOS?
}

José Joaquín Lunazzi

\section{Resumo}

Dois mil anos antes da chegada dos europeus, civilizações americanas contavam com espelhos capazes de fornecer boa qualidade de imagem. Notadamente, a Olmeca em Centroamérica, mas também a Cupisnique na América do Sul. Essa tecnologia óptica pode ter influenciado nos Maias e até nos Aztecas, embora saibamos que sua aplicação e difusão aparece como muito restrita. Porém ligada a relações de poder e representada em divindades. Por achados, pode-se ter uma ideia da técnica de sua realização mas, fora a presença junto aos esqueletos nas tumbas e a representação em murais e esculturas, não se tem mais elementos como para conhecer a maneira como teriam sido utilizados. Tentamos cobrir essa carência aplicando todas as relações lúdicas e de experimentação que podemos imaginar. Este estudo pode servir como orientação para ajudar a relacionar os espelhos com sua cultura.

Palavras-chave: espelhos arqueológicos, olmecas, culturas precolombinas, objetos cerimoniais, tecnologia primitiva

\section{Resumen}

Dos mil años antes de la llegada de los europeos, las civilizaciones americanas contaban com espejos de buena calidad de imagen. Particularmente, la Olmeca en Centroamérica, mas también la Cupisnique en América del Sur. Esa tecnología óptica puede haber influenciado a los Mayas y los Aztecas, aunque sabemos que su aplicación y difusión debe haber sido limitada. Mas, ligada a relaciones de poder y representada en divinidades. Analizando los ejemplares encontrados podemos tener una idea de como fueron realizados pero solo podemos deducir sobre su uso por su presencia en las tumbas y la representación en murales y esculturas. Intentamos cubrir esa carencia aplicando todas las relaciones lúdicas y de experimentación que podemos imaginar. Este estudio puede servir como orientación para ayudar a relacionar los espejos con su cultura.

Palabras clave: espejos arqueológicos, olmecas, culturas pre-colombianas, objetos ceremoniales, tecnologia primitiva 


\begin{abstract}
Two thousand years before the arrival of the europeans the american civilizations had good quality mirrors. Mainly the Olmeca culture in Central America and the Cupisnique in South America. That optical technology may had influence in the Maya and Aztec culture, although we know that its application and popularisation was very limited. It was linked to power relationships and represented with divinities. By analyzing the findings it is possible to derive how were made, but we can only know about his use by his presence at murals and sculptures. We try to discover it by applying all the experimentation we can conceive, hoping to help in relating the mirrors to its culture.
\end{abstract}

Keywords: archaeological mirrors, Olmecs, pre-columbian cultures, ceremonial objects, primitive technologies

\title{
1. Introdução
}

A presença de elementos brilhantes se nota até em nossos dias nas vestimentas de uso especial, festivo ou ceremonial, nos povos da América Latina. O uso de elementos ópticos refletivos são evidência de uma cultura sofisticada. Tem sido comprovado em antigas culturas como a egípcia, chinesa e grega, p.ex. Em um trabalho anterior (Lunazzi 2013) temos descrito a presença de espelhos em culturas americanas como a dos olmecas e cupisniques (achados no México e no Perú, respectivamente), faremos agora uma classificação técnica sistemática das possibilidades de uso desses espelhos, em versão resumida de uma publicação a acontecer proximamente (Lunazzi 2015).

\section{Caracterização física dos espelhos}

Um elemento refletor é caracterizado pelo parâmetro chamado refletividade, que indica percentualmente quanto da luz que incide nele não o penetra nem atravessa, voltando para a região do espaço de onde veio fazendo ângulos simétricos a respeito da superfície. Como tratamos de elementos geradores de imagens de qualidade, desprezamos a dispersão, referida a luz que volta em outros ângulos que não o simétrico e poderia gerar um halo de luz indesejada.

Consideramos que os elementos são utilizados de maneira enfrentada, o que permite considerar o valor de refletividade na incidência perpendicular como aplicável a toda a luz de

\begin{tabular}{|l|l|l|l|l|l|l|}
\hline (C Rev. Arqueologia Pública & Campinas, SP & v.9 & No.(11) & p.134-146 & suplemento & ISSN 2237-8294 \\
\hline
\end{tabular}


uma imagem. Em outras palavras, a refletividade muda pouco quando os ângulos da luz são próximos da perpendicular. Trata-se de uma característica válida para todo material, um resultado explicável pela física. Na falta de componentes metálicas a refletividade é aproximadamente $4 \%$, como no vidro, mas com os minerais utilizados resulta de $20 \%$ (hematita) e até $60 \%$ (pirita), valores bem tabelados em geologia pois servem na identificação do minério.

Para minhas considerações, tenho utilizado experimentalmente espelhos com refletividade de $20 \%$, pois nunca vi um espelho de pirita, material que deve ser futuramente considerado (MELGAR). Como tamanho, considero também um valor próximo da média dos espelhos conhecidos: $12 \mathrm{~cm} \times 12 \mathrm{~cm}$.

\section{Possíveis usos que descrevemos}

1) Observar seu próprio rosto

2) Observar imagens simétricas direita-esquerda

3) Observar imagens simétricas acima-embaixo

4) Observar imagens em profundidade por trás do espelho.

5) Observar imagens em profundidade a frente do espelho.

6) Impressionar às pessoas com reflexos brilhantes como símbolo de conjugação divina.

7) Ofuscar a visão das pessoas visando a ter vantagem em uma luta.

8) Se comunicar por meio da luz.

9) Fazer fogo com finalidade prática ou como símbolo de conjugação divina.

10) Impressionar às pessoas incorporando o espelho ao corpo.

\section{1) Observar o próprio rosto}

É indubitável que as primeiras experiências ao se obter o polimento refletivo levaram a observar o próprio corpo, e em consequencia a face. Deduzimos que, pela pouca refletividade

\begin{tabular}{|l|l|l|l|l|l|l|}
\hline (C) Rev. Arqueologia Pública & Campinas, SP & v.9 & No.(11) & p.134-146 & suplemento & ISSN 2237-8294
\end{tabular}


dos espelhos de qualidade, isto se deu com a pessoa sendo iluminada pelo sol de maneira direta e quase frontal, com o espelho na direção do sol. O espelho, ficando assim na sombra, permite a melhor observação de seu reflexo. Pode-se descartar a possibilidade de se ver em situações de iluminação por tochas ou em dias nublados.

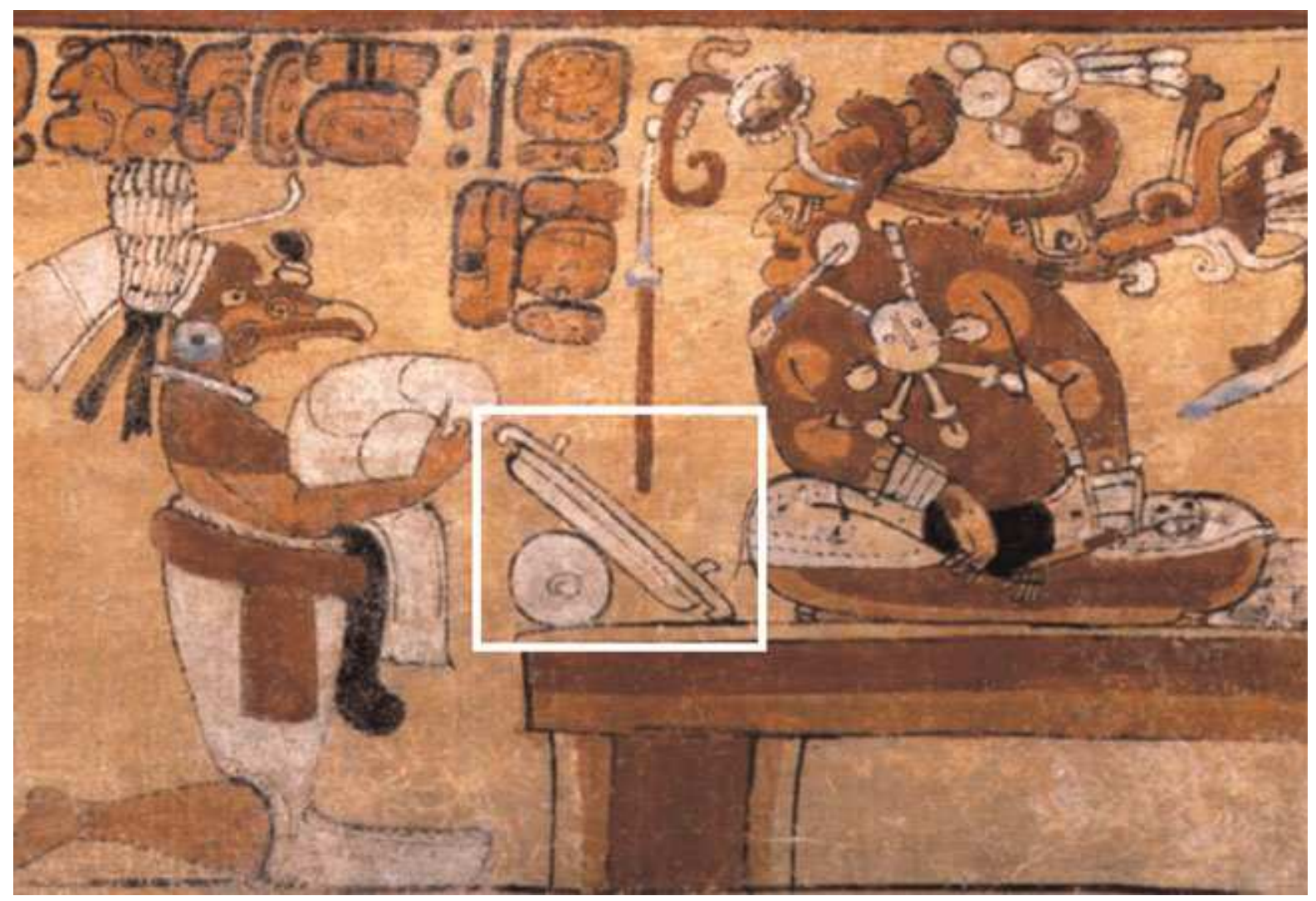

Figura 1: Representação de possível autocontemplação em espelho, em um vaso do período Clásico Maia (vase K5764) (Kerr 2006, Photograph K5764 @ Justin Kerr).(HEALY).

3.2) Observar imagens simétricas direita-esquerda

1 Pode ter demorado a percepção de que a imagem do espelho inverte lateralmente, por m exemplo o rosto. Mesmo a pessoa tendo alguma cicatriz, marca ou inscrição nele, é preciso uma observação bem criteriosa para notar isso. Já ao se colocar ao lado uma figura, a simetria

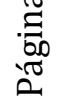
resulta sim evidente, mas lembrando a condição de iluminação melhor ser contra o sol, vemos que para se colocar ao lado um objeto iluminado, que o seria pelo sol, seria preciso cobrir ao

\begin{tabular}{|l|l|l|l|l|l|l|}
\hline (C) Rev. Arqueologia Pública & Campinas, SP & v.9 & No.(11) & p.134-146 & suplemento & ISSN 2237-8294
\end{tabular}


espelho da iluminação direta do sol. Com o próprio corpo, por exemplo. Se isto aconteceu, poderia haver figuras desenhadas lado a lado em simetria. Como de fato há, lembro ter visto um jaguar assim desenhado em uma parede em Teotihuacan. Não é fácil, porém, deduzir que a figura seja consequencia da observação com espelhos, pois figuras assim simétricas podem resultar da visão artística do desenhista,

\subsection{Observar imagens simétricas acima-embaixo}

Da mesma maneira que no item anterior, seria possível observar a inversão acontecendo desta outra maneira, ao se colocar um objeto ou figura por cima ou por baixo do espelho. Minha breve experiência no conhecimento da iconografia arqueológica não me permite dizer se haveria algum desenho assim. Mas posso sugerir a possibilidade de observação de objetos brilhantes, como o sol, a lua, ou as nuvens, com o espelho horizontalmente a frente do observador, na altura do nariz. Esses objetos do céu aparecem então embaixo, dentro da terra. Uma demonstração interessante utilizada atualmente no campo artístico e como experimento pedagógico (LUNAZZI 2009) nos leva a essa sugestão (Figura 2).

\begin{tabular}{|l|l|l|l|l|l|l}
\hline ( ) Rev. Arqueologia Pública & Campinas, SP & v.9 & No.(11) & p.134-146 & suplemento & ISSN 2237-8294 \\
\hline
\end{tabular}




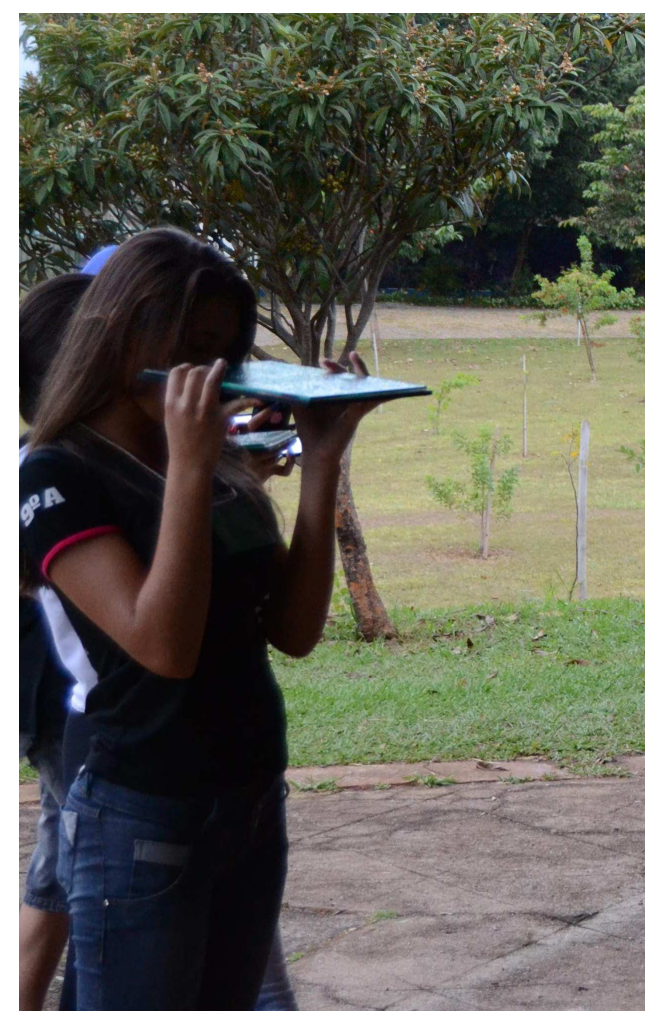

Figura 2: Pessoa experimentando a visão invertida do espaço, vendo o céu embaixo da terra por meio de um espelho.

Embora neste caso não temos uma visão em posição fixa por causa dos movimentos das mãos da pessoa que segura o espelho, se o mesmo estiver colocado em uma base, então sim, a ideia de ter os elementos do céu descidos para a terra resultaria possível. É uma situação assim que supomos possa ter sido utilizada pelos antigos.

3.4 Observar imagens em profundidade por trás do espelho.

No item anterior podemos pensar que o espelho tenha $12 \mathrm{~cm}$ ou mais de largura e comprimento, permitindo a visão nele com os dois olhos. A visão binocular representa a posição simétrica da imagem em profundidade, ou seja que o sol, a lua e as nuvens se vem a muita profundidade dentro do espelho, e bem dentro do chão, por baixo da terra, nos levando 
sentir a presença de um outro mundo em outra dimensão. No caso da imagem da lua em uma lagoa, índios acreditam que a lua esteja dentro da lagoa. Com um espelho devidamente posicionado na horizontal e fixo, seria possível mostrar a presença da lua em qualquer lugar, levando à ideia de uma conjugação com o divino, de um relacionamento entre o possuidor do espelho e os astros.

3.5 Observar imagens em profundidade a frente do espelho.

Os espelhos côncavos tem a propriedade de representar objetos claramente aparecendo a frente deles (LUNAZZI 2013). Para isto o objeto precisa estar a uma certa distância do espelho, simplesmente. O resultado é fantástico e fantasmagórico, transforma ao objeto em algo imaterial e espiritual. Bastaria colocar ao objeto de maneira oculta ao observador e invertido, para, pela imagem, fazer acreditar em uma presença puramente espiritual. Apenas devo lembrar da necessidade de se iluminar muito bem, o que deveria ser pelo sol. Não parece uma situação fácil, porém na iconografia de Tehotihuacan e outras tem-se cabeças de cobra aparecendo a frente de espelhos.

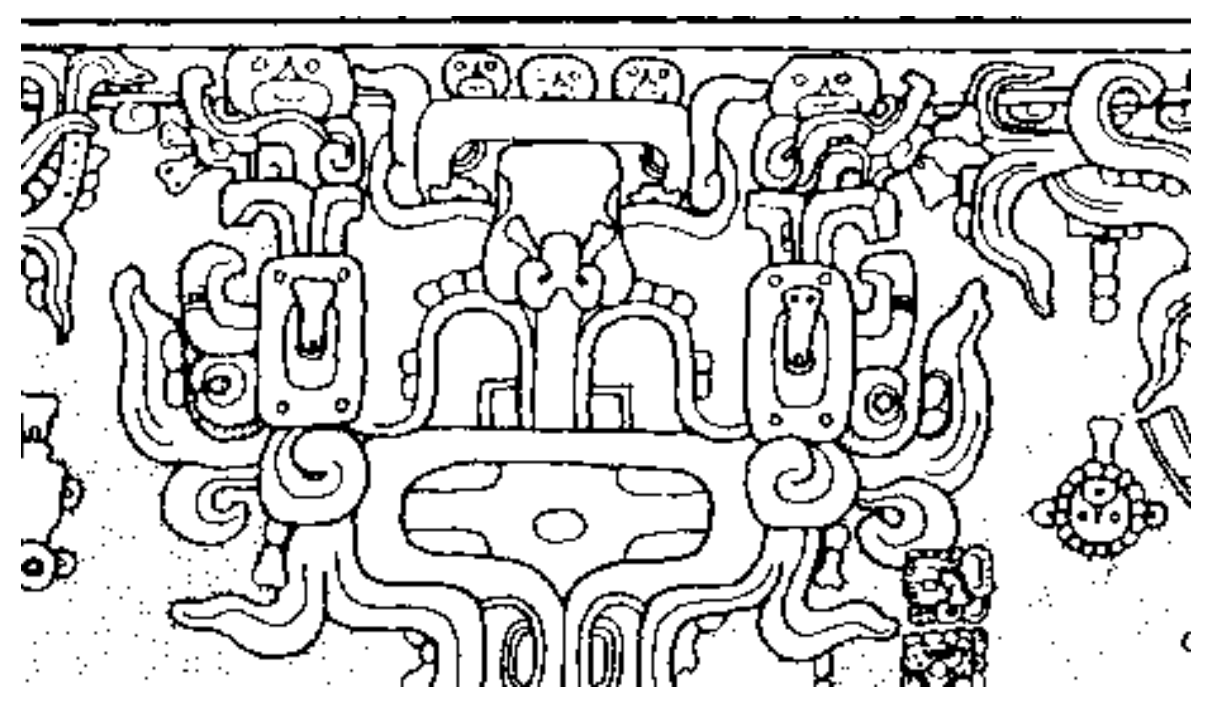

Figura 3: Na parte de cima, cabeças desenhadas invertidas em um mural (SCHELE) (CARLSON). Invertemos o detalhe para facilitar a observação, portanto trata-se de cabeças que aparecem não invertidas na figura.

\begin{tabular}{|l|l|l|l|l|l|l|}
\hline (C Rev. Arqueologia Pública & Campinas, SP & v.9 & No.(11) & p.134-146 & suplemento & ISSN 2237-8294 \\
\hline
\end{tabular}




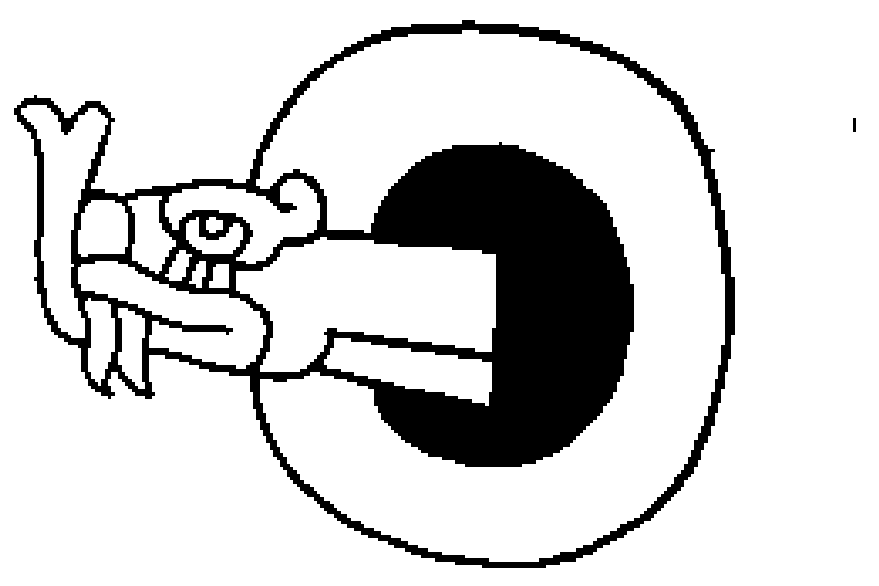

Figura 4: Cabeça de cobra representada saindo de um espelho (TAUBE).

3.6 Impressionar às pessoas com reflexos brilhantes como símbolo de conjugação divina.

Lançar reflexos aos olhos tem forte impacto psicológico e, por ser exclusividade de poucas pessoas, dada a raridade dos espelhos, poderia ter sido usado em cerimoniais e atos religiosos ao ar livre ou através de janelas ou portas.

3.7 Ofuscar a visão das pessoas visando a ter vantagem em uma luta.

O brilho do espelho com baixa refletividade ( $20 \%$ da hematita, caso dos olmecas e cupisniques) não chegaria a causar um incômodo suficiente. Deveriam de todas maneiras ser espelhos planos, que é o caso dos cupisniques, para não aumentar a divergência do feixe, reduzindo seu brilho. Talvez com espelhos de pirita (como os maias, com refletividade de $60 \%$ ), mesmo sendo de pouca qualidade como para fazer imagens boas, o caso faria sentido. Senão, apenas o caráter divino que pudesse lhe ser atribuído, serviria pelo poder psicológico.

3.8 Se comunicar por meio da luz.

(C Rev. Arqueologia Pública

Campinas, SP

v.9 No.(11) p. $134-146$ suplemento 
É uma possibilidade indiscutível. O experimento que realizamos a 1,4 km de distância (LUNAZZI 2013) (LUNAZZI 2012) evidenciou que seria possível se comunicar a distâncias bem maiores. Embora se trate de tamanhos maiores, reflexos do sol em janelas de vidro simples podem ser vistos a quilômetros de distância. O tamanho maior não altera o brilho, $\mathrm{e}$ resulta mais certa ainda a comparação porque a refletividade de uma lâmina de vidro é de somente $8 \%$. A limitação resulta de não poder usar em dia nublado, e mesmo assim, em condições de posição que dependem da hora, mudando da manhã para a tarde.

3.9 Fazer fogo com finalidade prática ou como símbolo de conjugação divina.

É possível fazer fogo por meio de espelhos côncavos e materiais combustíveis apropriados (LUNAZZI 1995), porém seria o caso de conferir a utilidade prática disto, pois de noite ou em dias nublados não resulta possível, enquanto as técnicas de fazer fogo por atrito estariam sempre disponíveis. Já como aparentamento de contato divino, o efeito não apenas é perfeito como aparece na figura do deus asteca Tezcatlipoca, chamado "do espelho fumegante", tendo por um pé a um espelho de onde sai fumaça (Figura 5)

\begin{tabular}{|l|l|l|l|l|l|l}
\hline ( ) Rev. Arqueologia Pública & Campinas, SP & v.9 & No.(11) & p.134-146 & suplemento & ISSN 2237-8294 \\
\hline
\end{tabular}




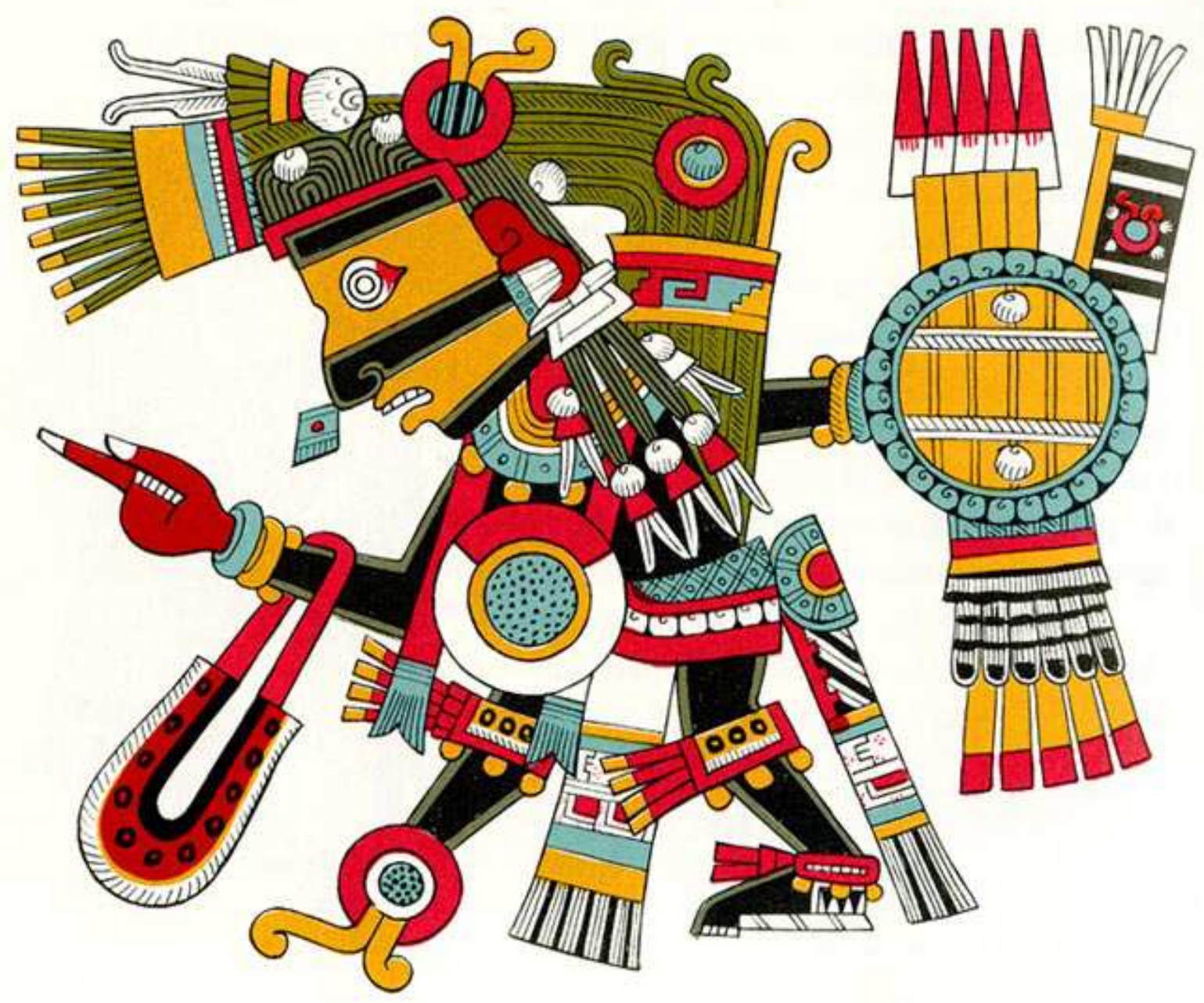

Figura 5: Representação do deus Tezcatlipoca (MEXICOLORE).

E existe descrito em literatura realizada logo após a conquista espanhola (DE LA VEGA) indicando uma cerimônia onde o fogo com o sol acontecia ligado à situação dos deuses com o povo.

$\underbrace{m}_{\substack{\pi \\ 0 \\ \pi \\ 0}}$

3.10 Impressionar às pessoas incorporando o espelho ao corpo.

(C) Rev. Arqueologia Pública

Campinas, SP

\begin{tabular}{|l|l|l|} 
v.9 & No.(11) & p.134-146
\end{tabular}

suplemento

ISSN 2237-8294 
Por qualquer um dos itens anteriores vemos que possuir um espelho, raridade tecnológica da época, constitui um símbolo de hierarquia, como de fato aparece na iconografia (CARLSON). E porque nos túmulos, que correspondem a pessoas nobres, os esqueletos aparecem com espelhos no pescoço, como colares.

\section{Conclusões}

São várias as possibilidades de utilização dos espelhos nas civilizações antigas. Não havendo documentos escritos a respeito na arqueologia americana, somente podemos nos basear em figuras, nos próprios espelhos encontrados, e em suposições de uso. Acreditamos que este texto possa ajudar a considerar situações de uso de espelhos na interpretação dos achados arqueológicos.

\section{Referências}

CARLSON, John B,"OImec Concave Iron Ore Mirrors: The Aesthetic of a Lithic Technology and the Lord of the Mirror", Dumbarton Oaks Conference on the Olmecs, Dumbarton Oaks, The University of Maryland, Washington, 117-147, na Fig. 35, "Tablet of the Foliated Cross", 1981.

DE LA VEGA, Garcilaso, “Comentarios Reales de los Incas”, Libro Sexto, capítulo XXII , Pedro Crasbeeck, Lisboa, 1609. Pode-se obter, por exemplo em:

<http://shemer.mslib.huji.ac.il/lib/W/ebooks/001531300.pdf $>$

HEALY, Paul F., Blainey, Marc G., "Ancient Maya Mosaic Mirrors: Function, Symbolism and Meaning", Ancient Mesoamerica, Cambridge University Press n. 22, p. 229-244, 2011. doi:10.1017/S0956536111000241 
LUNAZZI , José Joaquín "Haciendo fuego con espejo que simula un espejo arqueológico (olmeca)" Vídeo em: <https://www.youtube.com/watch?v=KsRRFw1bGZk> 1995.

. "La Nube: A maneira mais emocionante de experimentar espelhos planos", Caderno Catarinense de Ensino de Física, V26, N2, p.416-425, 2009.

<https://periodicos.ufsc.br/index.php/fisica/article/view/11357/10891>

Video em <https://www.youtube.com/watch?v=NXmNs6IX2Hg $>$

. "Projeto Comunicacoes Ópticas Pelo Sol Simulando Espelhos Arqueológicos Vídeo (quatrilingue) <https://www.youtube.com/watch?v=YMIPEkVOtOs>2012.

. "Óptica arqueológica como reforço da identidade americana e exemplo de desinformação", I Semana de Arqueologia da UNICAMP, Universidade Estadual de Campinas, São Paulo (Brasil), 2013. Disponível em:

$<$ http://www.nepam.unicamp.br/arqueologiapublica/revista/anais/arqueologia-e-tematicaindigena/PDFs/arquivo4.pdf>

Errata: na primeira línea da página 3, onde diz: "Chegando na sala onde tinha mais espelhos conferi que eram convexos" por vez de convexos deveria dizer côncavos.

. Capítulo 7 "On Brightness and Quality of Archaeological Mirrors found in the New World", no livro "Manufactured Light-Mirrors in the Mesoamerican Realm", Emiliano Gallaga, Marc G. Blainey eds., Colorado Press Dezembro 2015.

Hardcover ISBN: 978-1-60732-407-2 Brochura disponível em:

<http://www.upcolorado.com/university-press-of-colorado/item/2784-manufactured-light>

MELGAR, Emiliano; GALLAGA, Emiliano; SOLIS, Reyna, La pirita y su manufactura: análisis de cuatro contextos mesoamericanos. Estud. cult. maya, México , v. 43, 2014 . Disponível em $<$ http://www.scielo.org.mx/scielo.php?script=sci_arttext\&pid=S0185-

$25742014000100002 \&$ Ing=es\&nrm=iso $>$ accedido en 29 jun. 2015.

\section{MEXICOLORE}

(S) Página sobre simpósio acontecido na Inglaterra sobre o deus Tezcatlipoca, disponível em: 
SCHELE, Linda (1975) desenho de murais do Templo da Cruz Foliada. Disponível em: $<$ http://www.ancientamericas.org/collection/aa010859>

TAUBE, Karl A., "The Iconography of Mirrors at Teotihuacan" in "Art, Ideology and the City of Teotihuacan" , J.C. Berlo ed., 169-204, Dumbarton Oaks Research Library and Collection, Washington. Fig. 22, 1993. 